\title{
Fractional Rider and Multi-Kernel-Based Spherical SVM for Low Resolution Face Recognition
}

\author{
Renjith Thomas \\ GISAT \\ Kottayam, Kerala, India \\ renjiththomas80@gmail.com
}

\author{
Dr. MJS. Rangachar \\ Department of School of Electrical Sciences \\ Hindustan University \\ Chennai,Tamil Nadu, India
}

\begin{abstract}
Face recognition is a unique feature for recognizing the individual in the biometric system and is advantageous since face recognition is a non-contact process. However, biometric recognition is ineffective due to the low-resolution images, wanting the need for the effective recognition system. Accordingly, this research concentrates on developing an effective face recognition strategy using low and high-resolution images. Initially, the input low-resolution images are preprocessed for enhancing the image contrast and subjected to the generation of the high-resolution image. Then, the feature extraction using the GWTM process presents the texture features that facilitate effective recognition using the spherical Support Vector Machine (SVM) that works using the multiple kernel function. In the GWTM process, proposed fractionalROA is engaged in the optimal fusion of the features acquired from the wavelet, Linear Binary Patterns (LBP), and Gabor filter. The analysis of the recognition method is initiated based on the metrics, such as False Alarm Rate (FAR), False Rejection Ratio (FRR), and accuracy. The proposed fractional-ROA-based face recognition acquires the maximal accuracy, and minimal FRR and FAR of 0.98, 0.0123, and 0.0017, respectively.
\end{abstract}

Keywords: face recognition, optimization, support vector machine, machine learning, linear binary patterns

\section{Introduction}

Face recognition is one of the most popular biometric surveillance systems as a result of its uniqueness and minimal intrusive nature. Face recognition is a process of recognizing the face of the person through the extraction of significant information from the individual face image. The face recognition concept finds its valuable application in the areas of fusion, surveillance monitoring, security authentication, national security, and defense. However, the recognition rate is dependent on the classification accuracy of the classifier [9]. There are so many algorithms in the literature, where the vector is established from the face image, but the structural information is lost. Moreover, the details of the image are acquired to which the dimensional reduction techniques are applied in order to minimize the dimension of the features. These aforementioned feature and reduction mechanisms correspond to the classification accuracy. Some of the reduction mechanisms include the Principal Component Analysis (PCA) [12] and Linear Discrimination Analysis (LDA) [11].

On the other hand, the recognition is based on the classifiers and one of the earliest classifier employed is the RBF (Radial Basis Function) Neural Network, which comprises of three layers, such as input, hidden, and output layers. At the point of classification, the data is trained as two phases. Additionally, a SVM [13] plays a prominent role in face recognition [6]. The research concentrates in establishing the face recognition strategy based on the low-resolution images. Initially, the low-resolution image is pre-processed to facilitate the effective generation of the high-resolution image using the regression model. Then, the features are extracted from the low and the high-resolution images using the (Gabor filter + wavelet + texture) GWTM process that involves the generation of the wavelet features, LBP features, and Gabor features. Finally, all the three features that are extracted from both the high and low-resolution images are fused together to present the feature vector, where the feature fusion is facilitated using the proposed fractional ROA. Finally, the features are fed to the spherical SVM that classifies the individuals, promoting effective recognition.

The major contribution of the research is given as follows: 
The feature fusion is performed using the proposed fractional-ROA, which is the integration of the fractional concept in the standard ROA. The features are extracted from the low and the high resolution features individually and is fused together based on the proposed optimization

The paper is organized as: section 2 deliberates the motivation behind the research and section 3 structures the effective face recognition strategy. The results and discussion of the methods are presented in section 4 and finally, section 5 concludes the research.

\section{Literature Review}

In this section, the review of the existing methods is presented with the merits and demerits of the methods along with the major challenges of the research. Lu et al.[9] used the spare representation coding for the recognition process, where the features from the Gabor filter, LBP, and fisher criterion represented the feature vector. The face recognition rate was higher in the existing uncontrolled scenarios and the pose variation along with the illumination was taken into account. However, the performance degraded as a result of the interference. Wen and Wen [10] employed an algorithm based on the Improved Minimum Squared Error (IMSE), which was applied both for the original and the mirror images. The accuracy of face recognition was effective for the limited training samples with relevance to the poses. M. Jian and K. Lam [14] proposed a model on the basis of the on Singular Value Decomposition (SVD) for performing both face hallucination and recognition simultaneously. Conventionally, Low- Resolution face recognition was performed by super-resolving the Low- Resolution input face first, and subsequently performing face recognition to recognize the input face. T. Lu et al [15] presented a novel semi-coupled dictionary learning strategy to promote discriminative and representative abilities for face recognition and Super-Resolution (SR) simultaneously by relaxing coupled dictionary learning. Specifically, semi-coupled locality-constrained was used to enhance the consistency among Very Low-Resolution (VLR) and High-Resolution (HR) local manifold geometries, thus overcoming the negative effects of one-to-many mapping. However, the efficiency was affected in the case of the large-sized training samples. The challenges of the research were listed below: recognition accuracy is the major challenge that needs to be addressed using effective classifiers. Moreover, an effective means to handle the low-resolution image is required. Additionally, effective features for enhancing classification accuracy are required.

\section{Face Recognition using the Proposed Fractional Rider Optimization}

The ultimate aim of the face recognition strategy is to recognize the humans using the faces, which forms the major hectic challenge in the security applications in case of the low-resolution videos. Normally, the security concerns employ the surveillance videos for recognition the individuals, which may be of low resolution that insisted towards the generation of the method that better acknowledges the person using the low-resolution images. In this context, fractional ROA is employed for the generation of the highresolution image along with the spherical SVM that uses the multi-kernel functions. Following are the steps employed for recognition of the person: pre-processing, GWTM strategy for the generation of features, and recognition using the spherical SVM. Let us consider an image I of the size $[\mathrm{x} \times \mathrm{y}]$ taken from the database D. Initially, the input image is pre-processed in order to facilitate the extraction of the interested regions for further processing associated with the recognition process. Then, the kernel regression method is applied for the generation of the super-resolution image such that the lowresolution pixels are enhanced. Then, the features are extracted from the low resolution and the highresolution images such that effective recognition using spherical SVM is presented. In the feature fusion step of GWTM, the features are fused optimally for which the proposed fractional-ROA is employed. Fig. 1 shows the block diagram of the proposed recognition approach. 


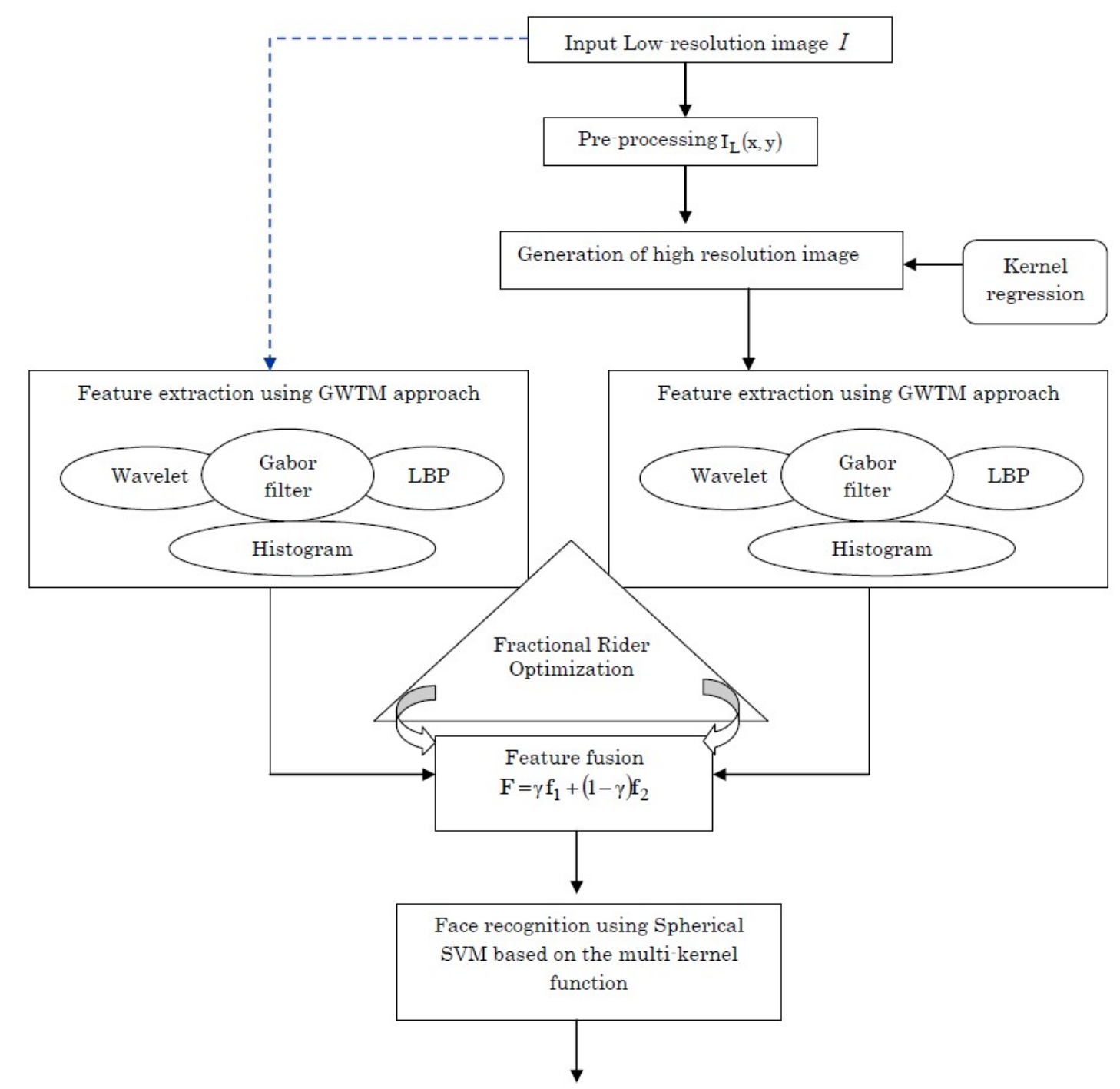

Fig. 1. Flow diagram of the face recognition approach based on fractional ROA and SVM classifier

\subsection{Pre-Processing}

In the first step of face recognition, the input images are pre-processed for the extraction of the region of interest of the face region for further processing such that the complexity associated with the feature extraction and classification accuracy of the classifier is enhanced. Moreover, the quality and reliability of the images are enhanced. Thus, the low-resolution image considered for further processing is denoted as, $\mathrm{I}_{\mathrm{L}}(\mathrm{x}, \mathrm{y})$.

\subsection{Kernel Regression to Acquire the High-Resolution Image}

The low dimension of the pixels in the low-resolution image insisted on the need for the generation of the high-resolution image, which is performed using the kernel regression. Thus, the high dimensional image possesses all the significant details of the image, which further enhances the recognition performance of the classifier. Initially, the kernel regression establishes the attribute window $\mathrm{X}(\mathrm{u}, \mathrm{v})$, where the scaling factor is defined based on the size of the window. On the other hand, the window carries the image pixels of poor intensity, which is later modified using the intensities of the neighboring pixels. Therefore, the pixels with low resolution are updated through multiplying the kernel matrix with the window considered. The formulation of the high-resolution image based on the kernel regression is represented as,

$$
I_{H}(x, y)=\frac{1}{g * h} \sum_{u=1 v=1}^{g} \sum^{h} X(u, v) * K(u, v)
$$


where, $X(\mathrm{u}, \mathrm{v})$ specifies the attribute window and $\mathrm{K}(\mathrm{u}, \mathrm{v})$ indicates the kernel window. Moreover, $\mathrm{g}$ and $\mathrm{h}$ corresponds to the rows and columns of the attribute window.

\subsection{Application of GWTM for Extracting the Features for Recognition}

The features are the effective means that facilitate recognition with higher recognition accuracy. Hence, feature extraction is performed using the GWTM process that comprises of the wavelet transforms, Gabor filters, and LBP. At first, the low and high-resolution image is subjected to the wavelet transforms, which extract the low and high-frequency components from the image. Then, the Gabor filter is applied that acquires the knowledge about the orientation and frequency of the low and highresolution images. Finally, the output of Gabor filter and wavelet transform, and the input image is subjected to the extraction of the texture features using the LBP descriptor. At last, the histogram of the LBP image is established. The feature extraction steps are common both for the input low resolution and high-resolution images such that the output of feature extraction using both the images are fused together to present the feature vector for recognition. The feature vector is represented as,

$$
\mathrm{F}=\gamma \mathrm{f}_{1}+(1-\gamma) \mathrm{f}_{2}
$$

where, $f_{1}$ represents the features extracted using the low-resolution image and $f_{2}$ symbolize the feature vector developed using the high-resolution image. More significantly, $\gamma$ is an optimal measure that fuses the features of low and high-resolution images in order to facilitate effective recognition. Therefore, the value of $\gamma$ is modeled optimally using the proposed fractional-ROA.

\subsection{Fractional Rider for Enabling the Optimal Feature Fusion}

In this section, the proposed fractional-ROA is presented that aims at computing the optimal value of $\gamma$ for effective feature fusion. Fractional-ROA is the integration of the fractional calculus [5] in the standard ROA [7], which is based on the imaginary ideas and thoughts, operating in the fictional computing platform unlike the other artificial computing and the nature-inspired algorithms. Thus, it is clear that the optimization steps of ROA are exhibited in the fractional-ROA such that the position update strategy of the riders is interpreted for computing the optimal values of $\gamma$. There are four-rider groups, such as bypass, attacker, overtaker, and follower and each of the groups aim at reaching the destination through adjusting their rider parameters, such as the steering angle of the rider vehicle, gear, accelerator, brake, and so on. The merits of ROA, such as the avoidance of the local optimal convergence, faster convergence to the global solution, selection of the optimal dimensional area, and exploring the search speed are facilitated using the individual rider groups. However, the ability to render an effective solution based on the historical records is a challenge that is ensured using the fractional calculus. Therefore, the standard update rule of overtaker is modified using the fractional calculus that would render effective convergence to the best solution.

3.4.1 Derivation for the update rule for the proposed fractional-ROA: In the proposed fractionalROA, the update rule of the overtaker is modified using fractional calculus. Generally, the overtaker is the one, who overtakes the leading rider to reach the destination. It is evident that the leading rider is the rider with the maximal success rate, which is the distance measure that symbolizes that the rider is near to the destination. Thus, the overtaker updates the position based on the following Eq:

$$
\mathrm{P}_{\mathrm{a}, \mathrm{b}}^{\text {over }}(\tau+1)=\mathrm{P}_{\mathrm{a}, \mathrm{b}}(\tau)+\left[\partial_{\mathrm{a}}^{\tau} * \mathrm{P}_{1, \mathrm{~b}}\right]
$$

where, $\mathrm{P}_{\mathrm{a}, \mathrm{b}}^{\text {over }}(\tau+1)$ is the new position of the rider $\mathrm{a}$ at $\mathrm{b}^{\text {th }}$ coordinate, $\mathrm{P}_{\mathrm{a}, \mathrm{b}}(\tau)$ is the position of the rider a at iteration $\tau$ or the previous position, $\partial_{\mathrm{a}}^{\tau}$ is the directional indicator of the rider a at iteration $\tau$, and $\mathrm{P}_{1, \mathrm{~b}}$ is the position of the leading rider. For approximation, the above Eq is rewritten as,

$$
\mathrm{P}_{\mathrm{a}, \mathrm{b}}^{\text {over }}(\tau+1) \quad \mathrm{P}_{\mathrm{a}, \mathrm{b}}(\tau)=\left[\partial_{\mathrm{a}}^{\tau} * \mathrm{P}_{1, \mathrm{~b}}\right]
$$

In the above $\mathrm{Eq}$, the fractional calculus is integrated as follows:

$$
\begin{gathered}
F^{\beta}\left[\mathrm{P}_{\mathrm{a}, \mathrm{b}}^{\text {over }}(\tau+1)\right]=\left[\partial_{\mathrm{a}}^{\tau} * \mathrm{P}_{1, \mathrm{~b}}\right] \\
\left\{\mathrm{P}_{\mathrm{a}, \mathrm{b}}^{\text {over }}(\tau+1)-\beta \mathrm{P}_{\mathrm{a}, \mathrm{b}}(\tau)-\frac{1}{2} * \beta *\left[\mathrm{P}_{\mathrm{a}, \mathrm{b}}(\tau-1)\right]-\frac{1}{6} \beta(1-\beta)\left[\mathrm{P}_{\mathrm{a}, \mathrm{b}}(\tau-2)\right]-\frac{1}{24} \beta(1-\beta)(2-\beta)\left[\mathrm{P}_{\mathrm{a}, \mathrm{b}}(\tau-3)\right]\right\}=\left[\partial_{\mathrm{a}}^{\tau} * \mathrm{P}_{1, \mathrm{~b}}\right] \\
\mathrm{P}_{\mathrm{a}, \mathrm{b}}^{\text {over }}(\tau+1)=\beta \mathrm{P}_{\mathrm{a}, \mathrm{b}}(\tau)+\frac{1}{2} * \beta *\left[\mathrm{P}_{\mathrm{a}, \mathrm{b}}(\tau-1)\right]+\frac{1}{6} \beta(1-\beta)\left[\mathrm{P}_{\mathrm{a}, \mathrm{b}}(\tau-2)\right]+\frac{1}{24} \beta(1-\beta)(2-\beta)\left[\mathrm{P}_{\mathrm{a}, \mathrm{b}}(\tau-3)\right]+\left[\partial_{\mathrm{a}}^{\tau} * \mathrm{P}_{1, \mathrm{~b}}\right]
\end{gathered}
$$


where, $\beta$ is a fractional constant and $P_{a, b}(\tau),\left[P_{a, b}(\tau-1)\right], P_{a, b}(\tau-2)$, and $P_{a, b}(\tau-3)$ are the position of $\mathrm{a}^{\text {th }}$ rider at the previous instances of the $a^{\text {th }}$ rider. Thus, Eq. (7) is the update Eq of overtaker in the proposed fractional-ROA.

3.4.2 Optimization steps of Fractional-ROA: The algorithmic steps of the proposed algorithm are deliberated in the following steps:

a) Initialization: As the first step of optimization, the rider parameters and the position of the riders are initialized. Let us assume that the position of the rider be denoted as

$$
\mathrm{P}_{\mathrm{a}, \mathrm{b}}(\tau) ; 1<\mathrm{a}<\mathrm{m} ; 1<\mathrm{b}<\mathrm{n}
$$

where, $\mathrm{m}$ and $\mathrm{n}$ are the total number of riders and coordinates, respectively. Among the total of $m_{\text {riders, }}$ there are four-rider groups, such as bypass, overtaker, attacker, and follower.

b) Computing the success rate: The success rate is a parameter that decides the leading rider or the best solution. The leading rider is the one near to the destination and the rider with the maximal success rate is declared as the winner of the race. Therefore, the success rate is formulated based on the distance between the position of the rider and the target position. The success rate is computed as,

$$
\mathrm{SR}=\frac{1}{[\mathrm{~J}-\mathrm{T}]}
$$

where, $\mathrm{T}$ describes the target. Therefore, it is evident that the success rate is inversely proportional to the distance as it increases with decreasing distance measure between the position of the rider and the destination.

c) Position Update of The Riders: In this step, the position of the riders is updated based on the position of the leading rider that is decided based on the success rate. The bypass rider bypasses the common path to reach the destination and the position is updated based on the following Eq:

$$
\mathrm{P}_{\mathrm{a}, \mathrm{j}}^{\text {bypass }}(\tau+1)=\lambda\left[\mathrm{P}_{\alpha, \mathrm{j}}(\tau) * \chi(\mathrm{j})+\mathrm{P}_{\mathrm{\eta}, \mathrm{j}}(\tau) *(1-\chi(\mathrm{j}))\right]
$$

where, $P_{\eta, j}(\tau)$ and $P_{\alpha, j}(\tau)$ refers to the position of the rider. $\alpha$ and $\eta$ are the random numbers that acquire the value between 1 and $\mathrm{m} . \chi(\mathrm{j})$ symbolize the random value between 0 and 1 , which is of size $[1 \times \mathrm{n}]$. It is clear from the above Eq that the bypass rider takes his/her own way to update the position irrespective to the position of the leading rider. At the same time, the follower updates the position following the position of the leading rider, which is modeled as,

$$
\mathrm{P}_{\mathrm{a}, \mathrm{b}}^{\text {follower }}(\tau+1)=\mathrm{P}_{1, \mathrm{~b}}+\left[\cos \mathrm{t}_{\mathrm{a}, \mathrm{b}}(\tau) * \mathrm{P}_{1, \mathrm{~b}} * \mathrm{~d}_{\mathrm{a}}^{\tau}\right]
$$

where, $t_{a, b}(\tau)$ refers to the steering angle of the $a^{\text {th }}$ rider at a time $\tau$, and $d_{a}^{\tau}$ specify the distance to be covered by the $\mathrm{a}^{\text {th }}$ rider. The follower follows the leading rider and his/her own steering angle to change the position in order to reach the destination. Furthermore, the overtaker updates the position based on the modified Eq presented in Eq. (7). The attacker updates the position based on the following Eq:

$$
\mathrm{P}_{\mathrm{a}, \mathrm{b}}^{\mathrm{atacker}}(\tau+1)=\mathrm{P}_{1, \mathrm{~b}}+\left[\cos _{\mathrm{a}, \mathrm{b}}(\tau) * \mathrm{P}_{\mathrm{l}, \mathrm{b}} * \mathrm{~d}_{\mathrm{a}}^{\tau}\right]
$$

It is clear from the above Eq that the position of the attacker is the same as that of the follower.

d) Finding the success rate: The success rate is evaluated for all the riders after the update of the position, which means that the leading rider need not be the same all the time as all the riders are engaged in reaching the destination first.

e) Rider parameter update: Once the leading rider is declared, the rider parameters, including all the vehicle parameters and activity counter are updated.

f) Riding Off time: The iterations are repeated for the maximal number of count until the best solution is determined.

\subsection{Multi-Kernel-Based Spherical SVM for Face Recognition}

The feature vector is presented to the recognition module, where the face recognition is performed based on the spherical SVM with the multikernel function [6]. The recognition is found to be effective using the classifier and the considered features enhance the classifier accuracy. 


\section{Results and Discussions}

In this section, the results and discussion of the recognition method developed based on the fractionalROA are presented, where the analysis is progressed based on the performance measures, such as accuracy, FAR, and FRR.

\subsection{Experimental Setup}

The face recognition strategy using the optimization is implemented in MATLAB that is installed in the PC with Intel core and Windows 8 operating system. The database considered for the implementation is the CVL database [3], which consists of 114 individual images with seven different spatial locations for the individuals. In this research, four samples are considered for the analysis purpose.

\subsection{Performance Measures}

The metrics considered for evaluating the methods include the accuracy, FRR, and FAR, which corresponds to the exactness of the face recognition strategy. The measure accuracy is the measure that evaluates the accurateness of the method in effectively recognizing the individuals using the lowresolution face images. On the other hand, FAR and FRR are the error measures that read the measure of missed recognition. The formula for evaluating the accuracy, FAR, and FRR is given by,

$$
\begin{aligned}
& \text { Accuracy }=\frac{\mathrm{T}^{+}+\mathrm{T}^{-}}{\mathrm{T}^{+}+\mathrm{T}^{-}+\mathrm{F}^{+}+\mathrm{F}^{-}} \\
& \text {far }=1-\text { specificity } \\
& \text { far }=1-\left[\frac{\mathrm{T}^{-}}{\mathrm{T}^{-}+\mathrm{F}^{+}}\right] \\
& \text {frr }=\frac{\mathrm{F}^{-}}{\mathrm{F}^{-}+\mathrm{T}^{+}}
\end{aligned}
$$

FRR is the ability of the security system in verifying the authenticated person and is based on false negatives as shown above.

\subsection{Competing Methods}

The methods used for the comparison are: Gabor Ordinal Measure (GOM) [2], LBP [1], Local Gradient Patterns (LGP) [4], GWTM, and GWTM with multi-kernel [8].

\subsection{Comparative Analysis}

In this section, the comparative analysis of the methods are presented based on the performance measures with respect to training percentage and epsilon,

4.4.1 Analysis based on the accuracy of the methods: The analysis based on accuracy with respect to the percentage of training and epsilon is presented. Fig. 2 shows the analysis based on the accuracy measure. The analysis based on accuracy with respect to the training percentage is depicted in fig. 2 a). When the percentage of training is 10, the accuracy of the methods, GOM, LBP, LGP, GWTM, and fractional-ROA-based GWTM + multi kernel SVM is $0.42,0.5,0.57,0.65,0.72$, and 0.82 , respectively. It is noted that with the increase in the training percentage, the accuracy of the methods increases. However, the proposed method outperforms the existing methods with maximal accuracy.

The analysis based on accuracy with respect to the epsilon is depicted in fig. 2 b). When the Epsilon is 5, the accuracy of the methods, GOM, LBP, LGP, GWTM, and fractional-ROA-based GWTM + multikernel SVM is 0.72., 0.7598, 0.7898, 0.8415, 0.8698, and 0.9751, respectively. It is noted that with the increase in the value of epsilon, the accuracy of the methods increase. However, the proposed method outperforms the existing methods with the maximal accuracy. 


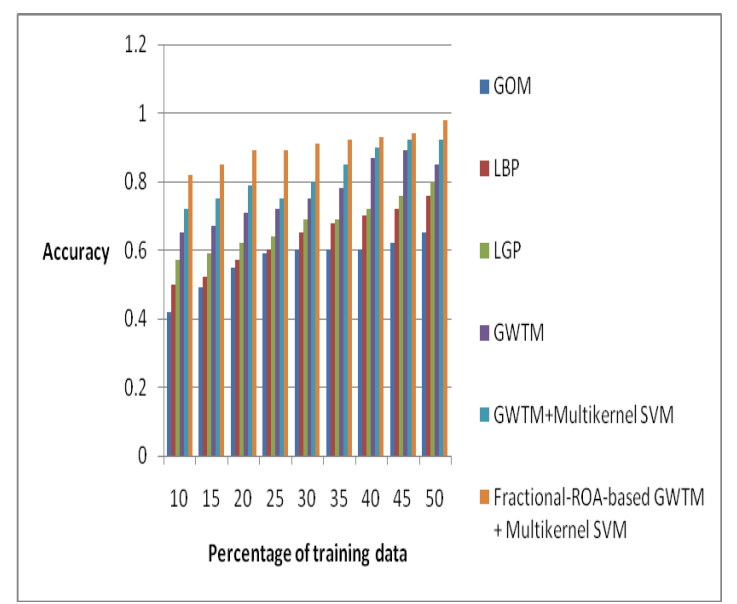

a)

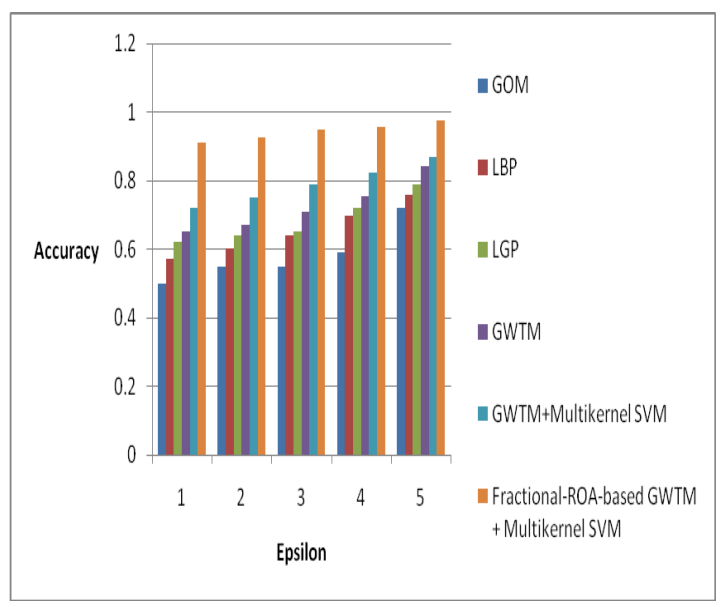

$\mathrm{b}$

Fig.2. Analysis based on accuracy, a) with respect to the training percentage, b) with respect to the epsilon

4.4.2 Analysis based on the FAR of the methods: The analysis based on FAR with respect to the percentage of training and epsilon is presented. Fig. 3 shows the analysis based on the FAR measure. The analysis based on FAR with respect to the training percentage is depicted in fig. $3 \mathrm{a}$ ). When the percentage of training is 10, the FAR of the methods, GOM, LBP, LGP, GWTM, and fractional-ROAbased GWTM + multikernel SVM is $0.5,0.7658,0.7546,0.74,0.6214$, and 0.5789, respectively. It is noted that with the increase in the training percentage, the FAR of the methods increase. However, the proposed method outperforms the existing methods with the maximal FAR.

The analysis based on FAR with respect to the epsilon is depicted in fig. 3 b). When the Epsilon is 5, the FAR of the methods, GOM, LBP, LGP, GWTM, and fractional-ROA-based GWTM + multikernel SVM is $0.123,0.1978,0.2456,0.1234,0.0023$, and 0.0017 , respectively. It is noted that with the increase in the value of epsilon, the FAR of the methods decreases. However, the proposed method outperforms the existing methods with minimal FAR.

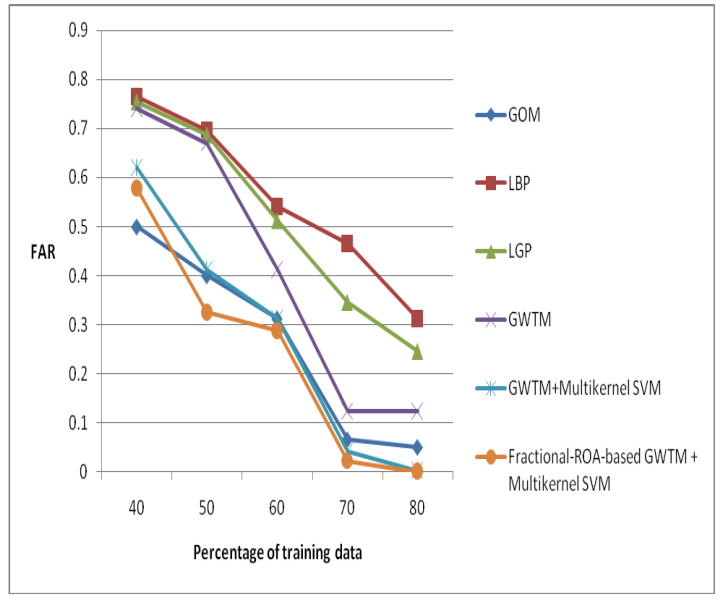

a)

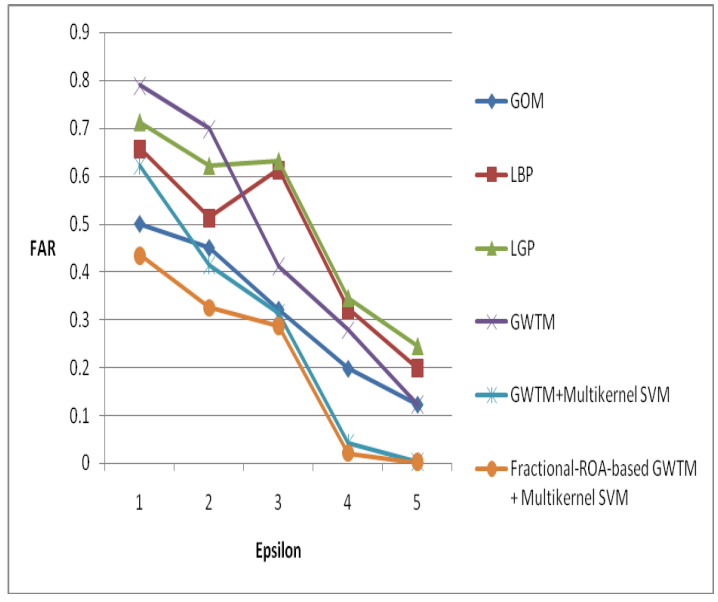

b)

Fig.3. Analysis based on FAR, a) with respect to the training percentage, b) with respect to the epsilon

4.4.3 Analysis based on the FRR of the methods: The analysis based on FRR with respect to the percentage of training and epsilon is presented. Fig. 4 shows the analysis based on the FRR measure. The analysis based on FRR with respect to the training percentage is depicted in fig. 4 a). When the percentage of training is 10, the FRR of the methods, GOM, LBP, LGP, GWTM, and fractional-ROAbased GWTM + multikernel SVM is 0.9997, 0.9891, 0.9888, 0.9877, 0.987, and 0.9, respectively. It is noted that with the increase in the training percentage, the FRR of the methods increase. However, the proposed method outperforms the existing methods with the maximal FRR.

The analysis based on FRR with respect to the epsilon is depicted in fig. 4 b). When the Epsilon is 5, the FRR of the methods, GOM, LBP, LGP, GWTM, and fractional-ROA-based GWTM + multikernel SVM is $0.1245,0.3214,0.4567,0.5678,0.3214$, and 0.0123 , respectively. It is noted that with the increase in 
the value of epsilon, the FRR of the methods decreases. However, the proposed method outperforms the existing methods with minimal FRR.

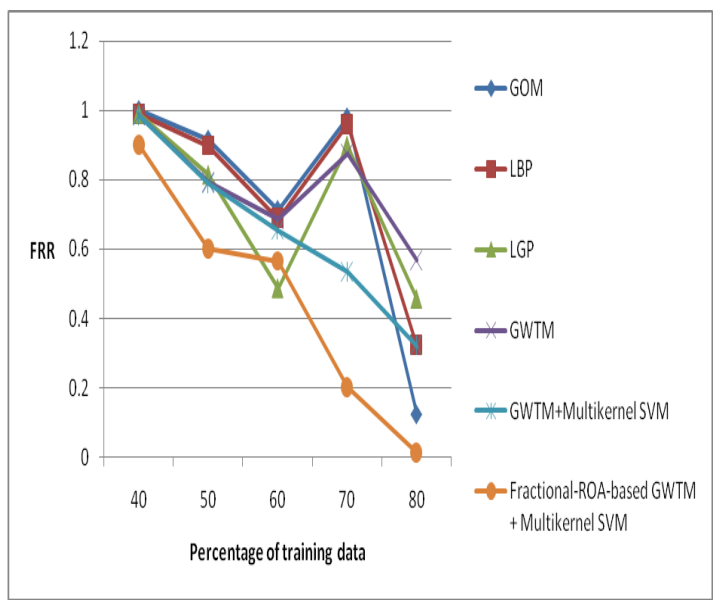

a)

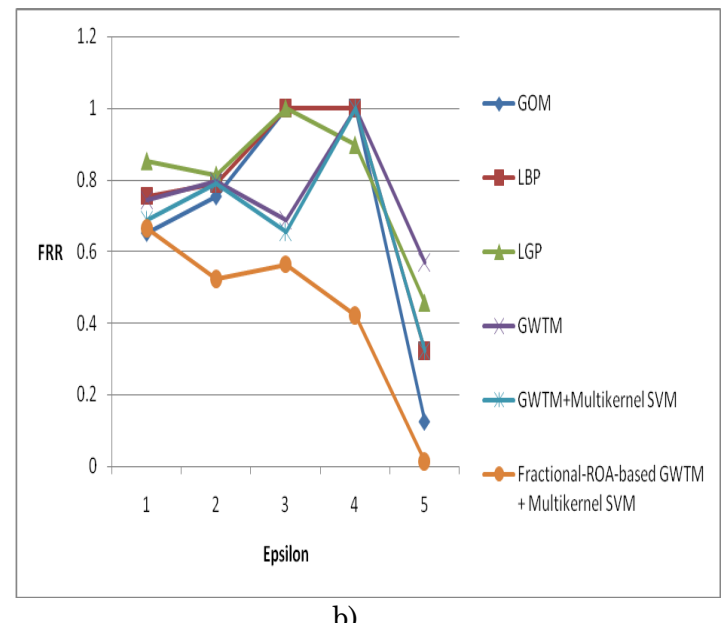

b)

Fig.4. Analysis Based On FRR, A) With Respect To The Training Percentage, B) With Respect To The Epsilon

\section{Conclusion}

The research concentrated on developing an effective face recognition strategy using low-resolution images. The security system using the face of the individuals suffers a lot during the authentication process due to the presence of the low-resolution images and hence, affects the recognition accuracy. Therefore, the paper developed a face recognition system using the spherical SVM with multi-kernel function. Initially, the low-resolution images are transformed as the high-resolution image using the regression model, which is then subjected to the recognition phase, where the features of both the low and high resolution images are presented. The features from the low and high resolution images are fused together using the fractional-ROA, which is proposed with the integration of the fractional concept in the ROA. The effectiveness of the proposed method of face recognition is facilitated using the CVL database, and the analysis is based on the measures, such as FRR, FAR, and accuracy. The FRR, FAR, and accuracy of the method is found to be $0.0123,0.0017$, and 0.98 , respectively. The future dimension of the research is based on any other hybrid optimizations and any other fusion features along with an effective classifier for recognition.

\section{Compliance with Ethical Standards}

Conflicts of interest: Authors declared that they have no conflict of interest.

Human participants: The conducted research follows the ethical standards and the authors ensured that they have not conducted any studies with human participants or animals.

\section{References}

[1] Md. Abdur Rahim and Md. Najmul Hossain, "Face recognition using local binary patterns (LBP)", Global J. Comput. Sci. Technol. Graphics Vis., Vol. 13, No. 4, 2013.

[2] Z. Chai and Z. Sun, "Gabor ordinal measures for face recognition", IEEE Trans. Inf. Forensics Security, Vol. 9, No. 1, 2014.

[3] CVL face database, Available at http://www.lrv.fri.uni-lj.si/facedb.html.

[4] M. Shahidul Islam, "Local gradient pattern - A novel feature representation for facial expression recognition", J. AI Data Mining, Vol. 2, 1, pp. 33-38, 2014.

[5] E. J. Solteiro Pires and J. A. Tenreiro Machado, "Particle swarm optimization with fractional-order velocity", Nonlinear Dynamics, Vol. 61, No. 1, pp. 295-301, 2010.

[6] R. Strack, V. Kecman, B. Strack and Q. Li, "Sphere support vector machines for large classi- cation tasks", Neurocomputing, Vol. 101, pp. 59-67, 2013.

[7] D. Binu and B. S. Kariappa, "RideNN: A New rider optimization algorithm-based neural network for fault diagnosis in analog circuits", IEEE Trans. Instrumentation and Measurement, Vol. 68, No. 1, pp. 2-26, 2019. 
[8] Renjith Thomas and M. J. S. Rangachar, " Fractional Bat and Multi-Kernel-Based Spherical SVM for Low Resolution Face Recognition", International Journal of Pattern Recognition and Artificial Intelligence, Vol. 31, No. 8, 2017.

[9] Z. Lu and L. Zhang, Face recognition algorithm based on discriminative dictionary learning and sparse representation, Neurocomputing Vol. 174, pp. 749-755, 2016.

[10] X. Wen and J. Wen, Improved the minimum squared error algorithm for face recognition by integrating original face images and the mirror images, Optik Vol. 127, No. 2, pp.883-889, 2016.

[11] J. Soldera, C. A. R. Behaine and J. Scharcanski, Customized orthogonal locality preserving projections with softmargin maximization for face recognition, IEEE Trans. Instrum. Meas. Vol. 64, No. 9, pp. 2417-2426, 2015.

[12] A. Deshmukh, S. Pawar and M. Joshi, Feature level fusion of face and fingerprint modalities using gabor filter bank, 2013 IEEE International Conference on Signal Processing, Computing and Control (ISPCC), Solan, pp. 1$5,2013$.

[13] X. Wu, W. Zuo, L. Lin, W. Jia and D. Zhang, "F-SVM: Combination of Feature Transformation and SVM Learning via Convex Relaxation," IEEE Transactions on Neural Networks and Learning Systems, Vol. 29 , no. 11, pp. 5185-5199, Nov. 2018.

[14] M. Jian and K. Lam, "Simultaneous Hallucination and Recognition of Low-Resolution Faces Based on Singular Value Decomposition," IEEE Transactions on Circuits and Systems for Video Technology, vol. 25, no. 11, pp. 1761-1772, Nov. 2015.

[15] T. Lu, X. Chen, Y. Zhang, C. Chen and Z. Xiong, "SLR: Semi-Coupled Locality Constrained Representation for Very Low Resolution Face Recognition and Super Resolution," IEEE Access, vol. 6, pp. 56269-56281, 2018. 